\title{
Pulmonary immune responses against SARS-CoV-2 infection: harmful or not?
}

\author{
A. Guillon ${ }^{1,2,3^{*}}$ (1) P. S. Hiemstra ${ }^{4}$ and M. Si-Tahar ${ }^{2,3}$
}

๑) 2020 Springer-Verlag GmbH Germany, part of Springer Nature

\begin{abstract}
Although our knowledge of the pulmonary immune response in patients with SARS-CoV-2 infection is still limited, intensivists will be offered to prescribe immunomodulatory interventions in patients with severe COVID-19 disease admitted to the ICU. Controlling the lung inflammatory response may be as important as targeting the virus, but the optimal timing and immune targets have not been clearly defined. Here, we summarize and discuss current knowledge on pulmonary immune responses against SARS-CoV-2 infection and its implication for decision-making processes at the ICU.
\end{abstract}

\section{The SARS-CoV-2 virus replicates in human airways but evades lung innate immune response}

COVID-19 autopsied lung studies ( $n=4$ patients) showed that ciliated cells of the proximal airway epithelium and alveolar cells (type I and II pneumocytes) were infected, but not goblet cells and submucosal glands [1]. The isolation of SARS-CoV-2 virus enabled experimental infections of human lung tissue, showing that SARS-CoV-2 replicates better than other coronaviruses, with a peak of viral replication reached in $48-72 \mathrm{~h}[2,3]$. Viral entry and cell infection trigger the host's immune response. However, immune evasion strategies are suspected by SARS$\mathrm{CoV}-2$ to alter host mucosal defenses. SARS-CoV-2 was capable of replicating in the human lungs while activating low levels of antiviral IFN (type I and III) and pro-inflammatory cytokines $[2,3]$. How SARS-CoV-2 virus subverts the first line of lung innate immunity is still an unanswered question, but coronaviruses are known for their ability to subvert innate immune response, including

*Correspondence: antoine.guillon@univ-tours.fr

${ }^{1}$ Intensive Care Unit, Tours University Hospital, 2 Bd Tonnellé, 37044 Tours Cedex 9, France

Full author information is available at the end of the article viral RNA sensing and signaling pathways involved in IFN secretion (reviewed in [4]). The limited activation of the innate immune response during the early phases of SARS-CoV-2 infection may facilitate its replication, but also explain the delayed clinical symptoms observed in most COVID-19 patients. This early, delayed immune activation may be followed in patients with severe disease by an imbalanced and overwhelming inflammatory response causing tissue injury and respiratory dysfunction (Fig. 1).

\section{The inflammatory response is mostly compartmentalized into the lung tissues (and is not a systemic cytokine storm)}

Immune responses with high levels of pro-inflammatory cytokines were described in the plasma of these patients, some of these cytokines being associated with the disease severity and progression [5]. This systemic inflammatory response was described as a "cytokine storm", but it is questionable whether this term is appropriate for the COVID-19 as mean circulating IL-6 levels often not exceeding $30 \mathrm{pg} / \mathrm{mL}$ [5-7]. In contrast, (i) an earlier report on human influenza infection showed mean IL-6 levels around $200 \mathrm{pg} / \mathrm{mL}$ [8]; (ii) a "cytokine storm" reported in a phase 1 trial (critically ill healthy volunteers after receiving anti-CD28 monoclonal antibody) was associated with more than $3000 \mathrm{pg} / \mathrm{mL}$ of IL-6 [9]. Therefore, the systemic IL-6 levels reported in many studies in critically ill COVID-19 patients are markedly lower than those that could be anticipated in case of a genuine "cytokine storm". In line with this view, most critically ill COVID-19 patients presented initially with isolated lung failure and not as in sepsis with multi-organ failure [10]. This clinical observation would, therefore, rather point to a local dysregulated inflammatory response that is largely restricted to the lungs. Consistently, comparative assessment 
No disease/mild disease

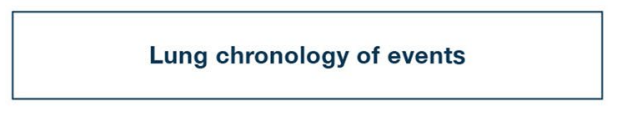

Host/SARS-CoV-2 interaction in lungs

Alveolar macrophage (phagocytosis of apoptotic epithelial cells)

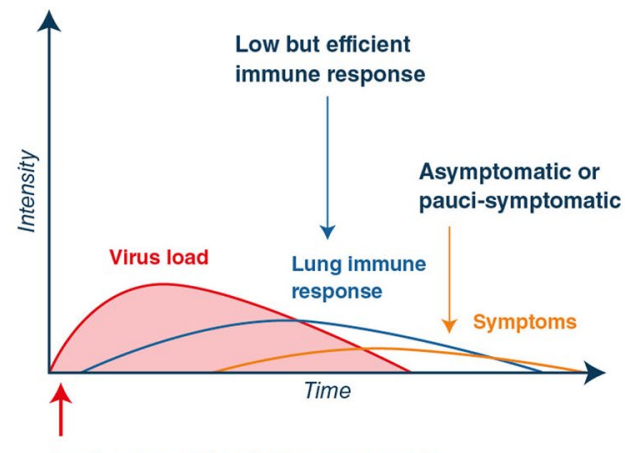

Replication with mild immune evasion

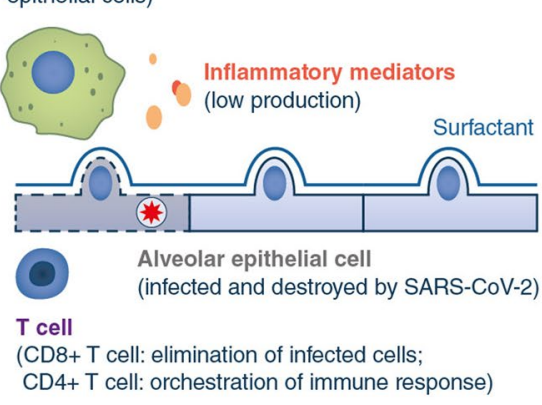

Immunosuppressive therapy

may prevent the worsening of the symptoms

and/or

may facilitate viral replicator

Critically ill COVID-19 patients
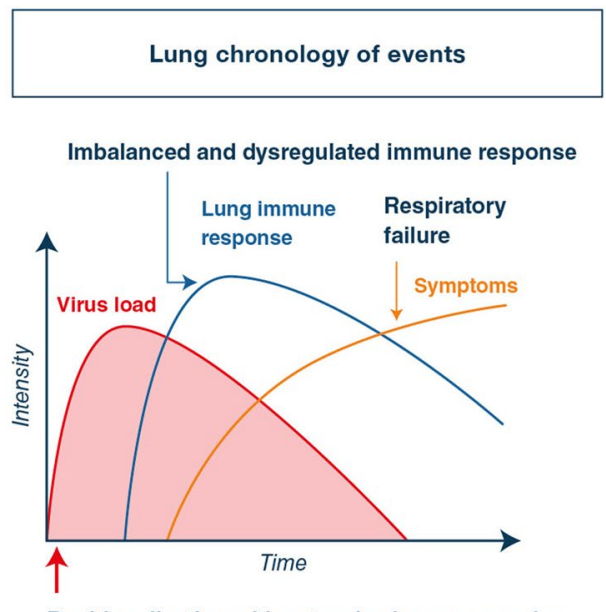

Host/SARS-CoV-2 interaction in lungs

Recruitment of monocyte-derived macrophages (activated and with pro-inflammatory phenotypes)

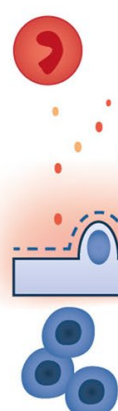

High production Damaged epithelial of inflammatory mediators * $2(2)$

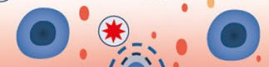
layer due to viral response

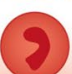

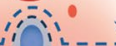

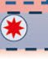

of lung epithelial (replication and release of virus)

Conventional and innate $T$ cells (recruited, activated and "exausted")

Fig. 1 Proposed model of host-pathogen interactions in the lungs of COVID-19 patients. SARS-CoV-2 virus is thought to initially infect the upper airways and reach the periphery of the lungs by microaspiration [1]. In those patients in which the virus has ended up infecting the peripheral lung, the majority of patients will likely clear the virus and have a limited inflammatory response with mild clinical disease (upper panels). However, insufficiently controlled SARS-CoV-2 replication due to suppression of antiviral defenses by the virus as well as to host susceptibility factors subsequently leads to a dysregulated inflammatory response which is probably mostly restricted to the lungs (lower panels). Clinical manifestations of COVID-19 are assumed to be explained by a combination of uncontrolled immune responses and virus-induced direct cytopathic effects (central, upper, and lower panels). Thus, immunotherapies should be considered with caution, if not given at the appropriate step of the disease as on one hand, they may efficiently target the host deleterious inflammatory response, but on the other hand, they may as well promote SARS-CoV-2 virus multiplication by inhibiting the host antiviral immune shield, thereby delaying virus clearance (left, upper, and lower panels)

of inflammatory mediators in the serum and respiratory fluids of the same patients $(n=30)$ demonstrated
100 - to 1000 -fold higher concentrations of IL- $1 \beta$ and IL-6 in the lung compartment [11]. 


\section{Lung hyperinflammation is characterized by infiltration of inflammatory monocyte-derived macrophages}

To further decipher the dysregulated inflammatory response in the lungs of COVID-19 patients, single-cell RNA sequencing was performed in bronchoalveolar lavage fluids from COVID-19 patients $(n=3$ severe patients, $n=3$ mild patients, and $n=8$ healthy controls). This analysis revealed a dysregulated balance of lung macrophage populations with a drastic increase in monocyte-derived macrophages [12]. Monocyte-derived macrophages were found to be activated and displayed pro-inflammatory phenotypes, such as enhanced transcription of STAT1, STAT2, and multiple IFN regulatory factors [12]. In line with this, a similar macrophage subtype was reported in lungs of euthanized non-human primates obtained at 7 days post-SARS-CoV-2 infection [13]. Previous studies examined the link between recruited inflammatory monocyte-macrophages and SARS-CoV-induced lung immunopathology [14]. Depletion of monocytemacrophages in SARS-CoV-infected mice resulted in increased survival, which is in line with a critical role for monocyte-macrophages in SARS-CoV-induced severe lung injury. Finally, post-mortem biopsies collected from COVID-19 patients confirmed the lung hyperinflammation with diffuse alveolar damage. Acute fibrinous and organizing pneumonitis (AFOP) was also a dominant histologic finding (series of six and ten patients) $[15,16]$. Since AFOP has been reported to be sensitive to corticosteroid treatment, this raises the question of a beneficial use of corticosteroids.

\section{The T lymphocyte immune response is altered in COVID-19 patients}

Because nearly $85 \%$ of critically ill patients with COVID-19 showed peripheral blood lymphopenia, lymphocyte immunophenotyping had rapidly emerged as a scientific priority. Preliminary flow cytometry assessment of blood immune cells demonstrated a reduction in absolute numbers of total $\mathrm{T}$ lymphocytes (CD4+, CD8+, and regulatory $\mathrm{T}$ cells) $[17,18]$. To explain the low lymphocyte counts observed in the COVID-19 patients, three main mechanisms are proposed: (i) direct lymphocyte destruction by SARSCoV-2 [19], (ii) host-mediated lymphopenia [20], and (iii) lymphocyte recruitment to lung tissue. A limitation of those studies is that they focused essentially on conventional $\mathrm{T}$ cells, whereas information on other $\mathrm{T}$ cells subsets in COVID-19 patients is barely available. Yet, the so-called innate $\mathrm{T}$ cells constitute an abundant component of the lung immune system and are considered potent immune orchestrators at barrier sites. We have recently reported a profound and persistent dysfunction of innate $\mathrm{T}$ cells in blood of critically ill COVID-19 patients and reported an unexpected infiltration and activation of these cells in the airways of these patients [11].

In conclusion, in the absence of effective antiviral treatments, there is so far only one mechanism that efficiently eradicates the SARS-CoV-2 from the host: its own immune response. Indeed, the large majority of patients recover without any curative treatment. A number of studies have explored strategies to dampen inflammatory responses, but the inflammatory response in viral pneumonia can be a double-edged sword (Fig. 1). Moreover, although elevated systemic levels of IL- 6 or IL-1 $\beta$ were found to be indicators of poor outcome in patients with severe COVID-19 with pneumonia, little is known about the local immune and inflammatory responses in lung tissue and their role in COVID-19 pneumonia. Inflammatory features that we are identifying so far in the systemic compartment could be either the tip of the iceberg or provide an inaccurate view of local inflammation in the lung compartment. There is an urgent need to understand the host-SARS-CoV-2 interaction within the lung tissue per se and to identify potential auto-amplification of inflammatory loops or other immunopathological process. Such knowledge is essential for informed clinical decision-making in the ICU.

\section{Author details \\ ${ }^{1}$ Intensive Care Unit, Tours University Hospital, 2 Bd Tonnellé, 37044 Tours Cedex 9, France. ${ }^{2}$ University of Tours, Tours, France. ${ }^{3}$ Research Center for Res- piratory Diseases, INSERM U1 100, Tours, France. ${ }^{4}$ Department of Pulmonology, Leiden University Medical Center, Leiden, The Netherlands.}

Author contributions

AG, PSH, and MST were involved in drafting the manuscript.

Compliance with ethical standards

Conflicts of interest

The authors declare that they have no conflict of interest.

Research involving Human Participants and/or Animals Not applicable

Informed consent

Not applicable

\section{Publisher's Note}

Springer Nature remains neutral with regard to jurisdictional claims in published maps and institutional affiliations.

Received: 30 April 2020 Accepted: 30 June 2020 Published online: 17 July 2020

References

1. Hou YJ, Okuda K, Edwards CE et al (2020) SARS-CoV-2 reverse genetics reveals a variable infection gradient in the respiratory tract. Cell. https:// doi.org/10.1016/j.cell.2020.05.042 
2. Hui KPY, Cheung M-C, Perera RAPM et al (2020) Tropism, replication competence, and innate immune responses of the coronavirus SARSCoV-2 in human respiratory tract and conjunctiva: an analysis in ex-vivo and in-vitro cultures. Lancet Respir Med. https://doi.org/10.1016/\$2213 $-2600(20) 30193-4$

3. Chu H, Chan JF-W, Wang Y et al (2020) Comparative replication and immune activation profiles of SARS-CoV-2 and SARS-CoV in human lungs: an ex vivo study with implications for the pathogenesis of COVID-19. Clin Infect Dis. https://doi.org/10.1093/cid/ciaa410

4. Kikkert M (2020) Innate immune evasion by human respiratory RNA viruses. J Innate Immun 12:4-20. https://doi.org/10.1159/000503030

5. Yang Y, Shen C, Li J et al (2020) Exuberant elevation of IP-10, MCP-3 and IL-1 ra during SARS-CoV-2 infection is associated with disease severity and fatal outcome. medRxiv. https://doi.org/10.1101/2020.03.02.20029975

6. Huang C, Wang Y, Li X et al (2020) Clinical features of patients infected with 2019 novel coronavirus in Wuhan, China. Lancet 395:497-506. https ://doi.org/10.1016/S0140-6736(20)30183-5

7. Qin C, Zhou L, Hu Z et al (2020) Dysregulation of immune response in patients with COVID-19 in Wuhan, China. Clin Infect Dis. https://doi. org/10.1093/cid/ciaa248

8. de Jong MD, Simmons CP, Thanh TT et al (2006) Fatal outcome of human influenza $A(\mathrm{H} 5 \mathrm{~N} 1)$ is associated with high viral load and hypercytokinemia. Nat Med 12:1203-1207. https://doi.org/10.1038/nm1477

9. Suntharalingam G, Perry MR, Ward S et al (2006) Cytokine storm in a phase 1 trial of the anti-CD28 monoclonal antibody TGN1412. N Engl J Med 355:1018-1028. https://doi.org/10.1056/NEJMoa063842

10. Ferreira M, Blin T, Collercandy N et al (2020) Critically ill SARS-CoV-2-infected patients are not stratified as sepsis by the qSOFA. Ann Intensive Care 10:43. https://doi.org/10.1186/s13613-020-00664-w

11. Jouan Y, Guillon A, Gonzalez L et al (2020) Functional alteration of innate T cells in critically ill Covid-19 patients. medRxiv. https://doi. org/10.1101/2020.05.03.20089300
12. Liao M, Liu Y, Yuan J et al (2020) The landscape of lung bronchoalveolar immune cells in COVID-19 revealed by single-cell RNA sequencing. medRxiv. https://doi.org/10.1101/2020.02.23.20026690

13. Bao L, Deng W, Gao H et al (2020) Reinfection could not occur in SARS-CoV-2 infected rhesus macaques. bioRxiv. https://doi. org/10.1101/2020.03.13.990226

14. Channappanavar R, Fehr AR, Vijay R et al (2016) Dysregulated type I interferon and inflammatory monocyte-macrophage responses cause lethal pneumonia in SARS-CoV-infected mice. Cell Host Microbe 19:181-193. https://doi.org/10.1016/j.chom.2016.01.007

15. Copin M-C, Parmentier E, Duburcq T et al (2020) Time to consider histologic pattern of lung injury to treat critically ill patients with COVID-19 infection. Intensive Care Med. https://doi.org/10.1007/s00134-020-06057

16. Schaller T, Hirschbühl K, Burkhardt K et al (2020) Postmortem examination of patients with COVID-19. JAMA. https://doi.org/10.1001/jama.2020.8907

17. Chen G, Wu D, Guo W et al (2020) Clinical and immunological features of severe and moderate coronavirus disease 2019. J Clin Investig. https:// doi.org/10.1172/JCI137244

18. Diao B, Wang C, Tan Y et al (2020) Reduction and functional exhaustion of T cells in patients with coronavirus disease 2019 (COVID-19). medRxiv. https://doi.org/10.1101/2020.02.18.20024364

19. Wang X, Xu W, Hu G et al (2020) SARS-CoV-2 infects T lymphocytes through its spike protein-mediated membrane fusion. Cell Mol Immunol. https://doi.org/10.1038/s41423-020-0424-9

20. Xiong Y, Liu Y, Cao L et al (2020) Transcriptomic characteristics of bronchoalveolar lavage fluid and peripheral blood mononuclear cells in COVID-19 patients. Emerg Microbes Infect 9:761-770. https://doi. org/10.1080/22221751.2020.1747363 